\title{
Entropic repulsion on a rarefied wall
}

\author{
L.R.G. Fontes ${ }^{1 \dagger}$ and M. Vachkovskaia ${ }^{2 \ddagger}$ and A. Yambartsev ${ }^{1 \S}$ \\ ${ }^{1}$ Instituto de Matemática e Estatística, Universidade de São Paulo, Rua do Matão 1010, CEP 05508-090, São Paulo \\ SP, Brazil \\ Irenatolime.usp.br, yambareime.usp.br \\ ${ }^{2}$ Instituto de Matemática, Estatistíca e Computação Científica, Universidade Estadual de Campinas, Caixa Postal \\ 6065, CEP 13083-970, Campinas SP, Brazil \\ marinaveime.unicamp.br
}

We consider the motion of a discrete $d$-dimensional random surface interacting by exclusion with a rarefied wall. The dynamics is given by the serial harness process. We prove that the process delocalizes iff the mean number of visits to the set of sites where the wall is present by some random walk is infinite. In case where there is a delocalization, bounds on its speed are obtained.

Keywords: harness process, surface dynamics, entropic repulsion, random environment

\section{Introduction and results}

The harnesses were introduced by Hammersley in [14] to model the long range correlations in the crystalline structures. The serial harness describes the evolution of a hypersurface of dimension $d$ embedded in a $(d+1)$-dimensional space. At each site $i \in \mathbb{Z}^{d}$ at time $n \geq 0$ we have the variable $Y_{n}(i)$ which denotes the height of a random surface at site $i$. The initial confi guration is the flat surface $Y_{0}(i)=0$ for all $i$. At each (discrete) moment $n \geq 0$ the height at each site is substituted by a weighted average of the heights at the previous moment plus a symmetric random variable (the noise).

Let $\mathcal{P}=\{p(i, j)\}_{i, j \in \mathbb{Z}^{d}}$ be a symmetric stochastic matrix which satisfi es $p(i, j)=p(0, j-i)=: p(j-$ $i)=p(i-j)$ (homogeneity) and $p(j)=0$ for all $|j|>v$ for some $v$ (fi niteness). Assume also that $\mathcal{P}$ is truly $d$-dimensional: $\left\{j \in \mathbb{Z}^{d}: p(j) \neq 0\right\}$ generates $\mathbb{Z}^{d}$.

Let $\mathcal{E}=\left(\varepsilon,\left(\varepsilon_{n}(i), i \in \mathbb{Z}^{d}\right), n \in \mathbb{Z}\right)$ be a family of i.i.d. integrable symmetric random variables with unbounded support.

The serial harness $\left(Y_{n}, n \geq 0\right)$ is the discrete time Markov process in $\mathbb{R}^{\mathbb{Z}^{d}}$ defi ned by

$$
Y_{n}(i)=\left\{\begin{array}{ccc}
0, & \text { if } & n=0 \\
\sum_{j \in \mathbb{Z}^{d}} p(i, j) Y_{n-1}(j)+\varepsilon_{n}(i), & \text { if } & n \geq 1
\end{array}\right.
$$

\footnotetext{
${ }^{\dagger}$ Partially supported by CNPq grants 300576/92-7 and 662177/96-7 (PRONEX) and FAPESP grant 99/11962-9.

¥Partially supported by FAPESP grant 99/11962-9 and FAEP grant 745/03.

$\S$ Supported by FAPESP grant 02/01501-9.
} 
So, the evolution can be written as

$$
Y_{n}=P Y_{n-1}+\varepsilon_{n},
$$

where $\varepsilon_{n}=\left(\varepsilon_{n}(i), i \in \mathbb{Z}^{d}\right)$. As the "noise variable" $\varepsilon$ is symmetric and thus has zero mean, we have that $\mathbb{E} Y_{n}(i)=0$ for all $i, n$. The weights $p(i, j)$ can be interpreted as transition probabilities of a random walk on $\mathbb{Z}^{d}$; denote by $p_{m}(i, j)$ its $m$-step transition probabilities. By homogeneity, $p_{m}(i, j)=p_{m}(0, j-i)=$ : $p_{m}(j-i)$. Iterating $(1)$, we get

$$
Y_{n}(i)=\sum_{r=1}^{n} \sum_{j \in \mathbb{Z}^{d}} p_{n-r}(i, j) \varepsilon_{r}(j) \stackrel{d}{=} \sum_{r=0}^{n-1} \sum_{j \in \mathbb{Z}^{d}} p_{r}(j) \varepsilon_{r}(j),
$$

for all $n \geq 1, i \in \mathbb{Z}^{d}$, where $\stackrel{d}{=}$ means equidistributed. In [14] it was shown that

$$
\mathbb{E}\left(Y_{n}(i)\right)^{2}=\sigma^{2} s(n),
$$

where $\sigma^{2}$ is the variance of $\varepsilon$ and

$$
s(n):=\sum_{r=0}^{n-1} \sum_{j \in \mathbb{Z}^{d}} p_{r}(j)^{2} .
$$

is the expected number of encounters up to time $n$ of two independent copies of a random walk starting at 0 with transition matrix $P$.

Since $s(n) \sim \sqrt{n}$ for $d=1, s(n) \sim \log n$ for $d=2$ and $s(n)$ is uniformly bounded in $n$ for $d \geq 3$ (see, for example, [19]), the surface delocalizes in dimensions $d \leq 2$ and stays localized in dimensions $d \geq 3$. Here $f(x) \sim g(x)$ means that $c_{1} g(x) \leq f(x) \leq c_{2} g(x)$ for all $x$ for some $0<c_{1}, c_{2}<\infty$.

The problem of entropic repulsion was extensively studied in statistic mechanics. The entropic repulsion for the Gaussian free fi eld was considered in $[2,3,7,8]$. For the Ising, SOS and related models the entropic repulsion was studied in $[4,5,6,9,12,15,17]$.

The serial harness interacting by exclusion with a wall located at the origin was studied in [11]. There, the following dynamics was considered. The wall process $\left(W_{n}, n \geq 0\right)$ is the Markov process in $\left(\mathbb{R}^{+}\right)^{\mathbb{Z}^{d}}$ defi ned by

$$
W_{n}(i)=\left\{\begin{array}{clc}
0, & \text { if } & n=0 \\
\left(\sum_{j \in \mathbb{Z}^{d}} p(i, j) W_{n-1}(j)+\varepsilon_{n}(i)\right)^{+}, & \text {if } & n \geq 1
\end{array}\right.
$$

for $i \in \mathbb{Z}^{d}$, where $a^{+}=a \vee 0=\max (a, 0)$; this can be rewritten as

$$
W_{n}=\left(P W_{n-1}+\varepsilon_{n}\right)^{+} .
$$

The most important results obtained there are the following two theorems.

Theorem 1. (Theorem 1.1 from [11]). (a) There is no nondegenerate invariant measure for the wall process $\left(W_{n}\right) ;\left(\right.$ b) $W_{n} \rightarrow \infty$ in probability; (c) $\mathbb{E} W_{n}(0) \rightarrow \infty$ as $n \rightarrow \infty ;$ (d) $\mathbb{E} W_{n}(0) / n \rightarrow 0$ as $n \rightarrow \infty$.

Theorem 2. (Theorem 1.2 from [11]). Let $F$ be the law of $\varepsilon, \bar{F}(x)=\mathbb{P}(\varepsilon>x)$ and define

$$
\begin{aligned}
\mathcal{L}_{\alpha}^{-} & :=\left\{F: \bar{F}(x) \leq c e^{-c^{\prime} x^{\alpha}}, x>0, \text { for some positive } c, c^{\prime}\right\} \\
\mathcal{L}_{\alpha}^{+} & :=\left\{F: \bar{F}(x) \geq c e^{-c^{\prime} x^{\alpha}}, x>0, \text { for some positive } c, c^{\prime}\right\}
\end{aligned}
$$


and

$$
\mathcal{L}_{\alpha}:=\mathcal{L}_{\alpha}^{-} \cap \mathcal{L}_{\alpha}^{+} .
$$

There exist constants $c$ and $C$ that may depend on the dimension such that

(i) for $d=1$ if $F \in \mathcal{L}_{1}^{-}$,

$$
c n^{1 / 4} \leq \mathbb{E} W_{n}(0) \leq C n^{1 / 4} \sqrt{\log n} ;
$$

(ii) for $d=2$, if $F \in \mathcal{L}_{\alpha}$, for some $\alpha \geq 1$,

$$
c(\log n)^{\frac{1}{\alpha} \vee \frac{1}{2}} \leq \mathbb{E} W_{n}(0) \leq C \log n ;
$$

(iii) for $d \geq 3$, if $F \in \mathcal{L}_{\alpha}$, for some $1 \leq \alpha \neq 1+d / 2$,

$$
c(\log n)^{\frac{1}{\alpha}} \leq \mathbb{E} W_{n}(0) \leq C(\log n)^{\frac{1}{\alpha} \vee \frac{2}{2+d}} ;
$$

(iv) for $d \geq 3$ if $F \in \mathcal{L}_{1+d / 2}$,

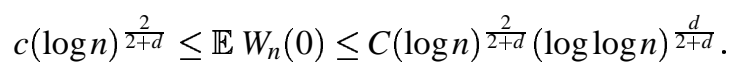

We note that in the case (ii) and (iii) above, the lower and upper bounds are of the same order in the case that $d \geq 3,1 \leq \alpha<1+d / 2$ (which includes the Gaussian case $\alpha=2$ for all such dimensions), and also in the case that $d=2, \alpha=1$.

The bound (slightly weaker than) (11) was proved in [10] for a one dimensional interface related to the phase separation line in the two dimensional Ising model at zero temperature.

$[2,3,7]$ considered the massless free fi eld (that is, centered Gaussian fi eld with covariance given by the Green functions of the SRW in $\mathbb{Z}^{d}$ ) in equilibrium and conditioned to stay positive in a large box. Among other things, the dependence of the height of the surface to the size of the box was studied and estimates with similar orders of magnitude to those of Theorem 2 were obtained. The massless free fi eld interacting with a wall of random height was studied in [1], and estimates similar to those in [2, 3, 7] for the wall with fi xed height case were obtained, showing in some cases an effect of the wall height distribution.

We next consider a modifi cation of the serial harness interacting with a wall, also in the direction of a variable wall. But the variability is in the presence or absence of the wall, always at fi xed height (if present), that is, we place a piece of flat wall located at height 0 at some sites of $\mathbb{Z}^{d}$, thus defining the environment

$$
W=\left\{i \in \mathbb{Z}^{d}: \text { there is a wall in } i\right\} .
$$

The set $W$ can be either random or deterministic. Then, with the environment fi xed, we start the serial harness which is not allowed to go below the wall (in the sites where the wall is present). So, the wall process $X_{n}^{W}(i), i \in \mathbb{Z}^{d}, n \geq 0$ is defi ned by

$$
X_{n}^{W}(i)=\left\{\begin{array}{cll}
0, & \text { if } & n=0, \\
\left(\sum_{j \in \mathbb{Z}^{d}} p(i, j) X_{n-1}^{W}(j)+\varepsilon_{n}(i)\right)^{+}, & \text {if } & n \geq 1 \text { and } i \in W, \\
\sum_{j \in \mathbb{Z}^{d}} p(i, j) X_{n-1}^{W}(j)+\varepsilon_{n}(i), & \text { if } & n \geq 1 \text { and } i \notin W .
\end{array}\right.
$$


Suppose that the set $W$ is such that either for all $i \in \mathbb{Z}^{d}$ the mean number of visits to the set $W$ by the random walk with transition matrix $\mathcal{P}$ starting from $i$ is infi nite, or there exists a constant $C$ such that for all $i \in \mathbb{Z}^{d}$ the mean number of visits to the set $W$ by the random walk with transition matrix $\mathcal{P}$ starting form $i$ is less than $C$. So, the following situation is impossible: there exists a sequence $i_{1}, i_{2}, \ldots \in \mathbb{Z}^{d}$ such that the mean number of visits to the set $W$ by the random walk with transition matrix $\mathcal{P}$ starting from $i_{n}$ is equal to $M_{n}$ and $M_{n} \rightarrow \infty$ as $n \rightarrow \infty$. This assumption is not very restrictive and it holds e.g. in the particular case considered in Theorems 4 and 5 .

We say that the process localizes (and that there occurs localization), if $\mathbb{E} X_{n}^{W}(i) \leq$ const for all $n$ and $i$, and delocalizes (there occurring delocalization) if there exists at least one site $i \in \overline{\mathbb{Z}}^{d}$ such that $\mathbb{E} X_{n}^{W}(i)$ is unbounded as $n \rightarrow \infty$ (the next theorem shows that those two alternatives cover in fact all the possible cases for which $W$ satisfi es the assumption on the latter paragraph). Our fi rst result is quite general.

Theorem 3. Under the above assumption on $W$, the process delocalizes iff the mean number of visits to the set $W$ by the random walk with transition matrix $\mathcal{P}$ is infinite.

We study in more detail the case when the wall is placed at each site $i \in \mathbb{Z}^{d}$ with probability $q_{i}$, independently of all other sites, where $q_{i}$ is radially symmetric, $q_{i}=q(\|i\|)$. We consider two cases: $q(x)=(x+1)^{-\beta}, \beta>0$ or $q(x)=\left(x^{2} \log ^{\gamma}(x+1)\right)^{-1}, \gamma>0$. If $q_{i}=1$ for all $i$, we get the process considered in [11]. It can be easily shown that all upper bounds obtained in [11] are valid for this modifi cation also. In this case the fact that the mean number of visits to the set $W$ is fi nite is equivalent to transience of $W($ see $[16,18])$.

In the case when $\mathbb{E} X_{n}^{W}(i)$ diverges, we are able to obtain lower bounds, which depend on the tail of the distribution of $\varepsilon$ and on $q(x)$, but we need a technical restriction on the transition matrix $\mathcal{P}$.

Theorem 4. Suppose that $p(0,0)=\delta>\frac{1}{2 d+1}$ and $p(0, i)=\frac{1-\delta}{2 d}$ if $\|i\|=1$. Let $d \geq 3$ and $q(x)=(x+1)^{-\beta}$, $\beta>0$.

(i) If $\mathbb{P}[\varepsilon>x] \sim e^{-x^{\alpha}}, \alpha>0$ and $\beta<2$, then for any i there exists $c=c(i, \beta)$ such that for all $n$

$$
\mathbb{E} X_{n}^{W}(i) \geq c(\log n)^{\frac{1}{\alpha}} .
$$

(ii) If $\mathbb{P}[\varepsilon>x] \sim e^{-x^{\alpha}}, \alpha>0$ and $\beta=2$, then for any i there exists $c=c(i)$ such that for all $n$

$$
\mathbb{E} X_{n}^{W}(i) \geq c(\log \log n)^{\frac{1}{\alpha}} .
$$

Note that in the case $(i)$ the lower bound is of the same order as in the process with $q_{i} \equiv 1$. If $\alpha<$ $1+d / 2$, then Theorems 2 and 4 give upper and lower bounds of the same order. So, the case when $q(x) \geq x^{-\beta}, \beta<2$, is similar to $q_{i} \equiv 1$ from the point of view of the repulsion speed.

Another remark is that under the assumption of Theorem 4, if $\beta>2$, then Theorem 3 implies localization, which together with the results of Theorem 4, establish a transition delocalization/localization at $\beta=2$.

A further point is that again under the assumption of Theorem 4, our better upper bound for case (ii) is by using the same bound as for the case $q_{i} \equiv 1$ of Theorem 2 . Thus we do not resolve the issue of whether or not there is a difference in repulsion speed (in leading order) between the cases treated in Theorems 2 and 4(ii). We can nevertheless establish a difference between the case of Theorem 2 and some of the ones treated in the following result. 
Theorem 5. Suppose that $p(0,0)=\delta>\frac{1}{2 d+1}$ and $p(0, i)=\frac{1-\delta}{2 d}$ if $\|i\|=1$. Let $d \geq 3$ and $q(x)=$ $\left(x^{2} \log ^{\gamma}(x+1)\right)^{-1}, \gamma>0$.

(i) If $\mathbb{P}[\varepsilon>x] \sim e^{-x^{\alpha}}, \alpha>0$ and $\gamma<1$, then

$$
c_{1}(i, \gamma)(\log \log n)^{\frac{1}{\alpha}} \leq \mathbb{E} X_{n}^{W}(i) \leq c_{2}(\log n)^{1-\gamma} .
$$

(ii) If $\mathbb{P}[\varepsilon>x] \sim e^{-x^{\alpha}}, \alpha>0$ and $\gamma=1$, then

$$
c_{1}(i)(\log \log \log n)^{\frac{1}{\alpha}} \leq \mathbb{E} X_{n}^{W}(i) \leq c_{2} \log \log n .
$$

From Theorems 2 and 5, when $\alpha \geq 1$ and $\gamma>(\alpha-1) / \alpha$, we can differentiate between the repulsion speeds of the two cases.

Note that in the framework of the hypotheses of Theorem 5, there is a delocalization/localization transition at $\gamma=1$, the localization upper bounds for the case $\gamma>1$ following from Theorem 3 .

Here we give main steps of the proofs. Full proofs will appear in [13].

\section{Main steps of proofs}

Main steps of the proof of Theorem 3. To prove that if the mean number of visit to $W$ is bounded, then there is localization we fi rst bound $X_{n}^{W}(0)$ from above by

$$
\sum_{j \in W} \sum_{k=0}^{n} S_{n-k}^{+}(j) p_{k}(0, j), \text { where } S_{n}(i)=\sum_{j \notin W} \sum_{k=1}^{n} p_{n-k}^{W}(i, j) \varepsilon_{k}(j)
$$

It can be easily seen that $\mathbb{E}\left(S_{n}^{+}(i) \mid W\right) \leq C$ uniformly in $W$ for all $n$ and $i$ for some $C>0$. Thus,

$$
\mathbb{E} X_{n}^{W}(0) \leq C \mathbb{E} \sum_{j \in W} \sum_{k=0}^{n} p_{k}(0, j) \leq c_{2} \sum_{k=1}^{n} k q(k)
$$

Note that the middle term of (20) is the mean number of visits to $W$ by time $n$ by the random walk with transition matrix $\mathcal{P}$. We thus get suffi ciency.

Suppose now that the process localizes, and thus $\mathbb{E} X_{n}^{W}(i)$ is uniformly bounded. This implies that there exists $K>0$ such that $\mathbb{P}\left[\mathcal{P} X_{n}^{W}(i)>K\right]<1 / 2$ for all $n$ and $i$. As $\varepsilon$ has infi nite support, $\mathbb{P}[\varepsilon<-K-1]>\delta$ for some $\delta>0$. Thence,

$$
\mathbb{E} X_{n}^{W}(i) \geq \mathbb{E}\left(\sum_{j \in \mathbb{Z}^{d}} p(i, j)\left(X_{n-1}^{W}(j)-Y_{n-1}(j)\right)\right)+\frac{\delta}{2} .
$$

Iterating, we get

$$
\mathbb{E} X_{n}^{W}(i) \geq \frac{\delta}{2} \sum_{k=0}^{n} \sum_{j \in W} p_{k}(i, j)
$$

and this yields necessity. 
Main steps of the proofs of Theorems 4 and 5. From Jensen's inequality, we get

$$
\mathbb{E} X_{n}^{W}(i) \geq \mathcal{P} \mathbb{E} X_{n-1}^{W}(i)+G\left(\mathcal{P} \mathbb{E} X_{n-1}^{W_{i}}(i)\right) q_{i},
$$

where $G(x)=\mathbb{E}\left(\varepsilon_{n}(i)-x\right)^{+}$and $W_{i}=W \cup\{i\}$. A coupling argument then yields

$$
\mathbb{E} X_{n}^{W_{i}}(j)-\mathbb{E} X_{n}^{W}(j) \leq C_{0}
$$

for some $C_{0}>0$, for all $n, i$, and $j$.

We next bound $\mathbb{E} X_{n}^{W}(i)$ from below by the following recursively defi ned family of variables:

$$
v_{n}(i)=\mathcal{P} \vee_{n-1}(i)+\tilde{G}\left(\mathcal{P} v_{n-1}(i)\right) q_{i},
$$

where $\tilde{G}(x)=G\left(x+C_{0}\right)$, and $v_{0}(i)=0$ for all $i$.

It then follows that

$$
\vee_{n}(j) \geq \sum_{k=1}^{n} \tilde{G}\left(\mathcal{P} v_{n}(j)\right) q\left(\mathbb{E}\left\|Z_{k}^{(i)}\right\|\right)
$$

if $i$ is such that $\|j\|<\|i\|-n$, where $Z_{k}^{(i)}$ is a random walk with transition matrix $\mathcal{P}$ starting at $i$.

We fi nally obtain

$$
v_{n}(j) \geq c^{\prime} \sum_{k=1}^{n} e^{-\left(\mathcal{P} v_{n}(j)+C_{0}\right)^{\alpha}}(\|i\|+k)^{-\frac{\beta}{2}}
$$

and (16-17) follow.

The upper bounds of Theorem 5 are immediate from (20)), and the lower bounds can be obtained as those of Theorem 4.

\section{References}

[1] D. Bertacchi, G. Giacomin (2002) Enhanced interface repulsion from quenched hard-wall randomness. Probab. Theory Relat. Fields 124, 487-516.

[2] E. Bolthausen, J.-D. Deuschel, G. Giacomin (2001) Entropic repulsion and the maximum of the two-dimensional harmonic crystal. Ann. Probab. 29 (4), 1670-1692.

[3] E. Bolthausen, J.-D. Deuschel, O. Zeitouni (1995) Entropic repulsion of the lattice free fi eld. Comm. Math. Phys. 170 (2), 417-443.

[4] J. Bricmont (1990) Random surfaces in statistical mechanics in Wetting phenomena. Proceedings of the Second Workshop held at the University of Mons, Mons, October 17-19, 1988. Edited by J. De Coninck and F. Dunlop. Lecture Notes in Physics 354. Springer-Verlag, Berlin.

[5] J. Bricmont, A. El Mellouki, J. Fröhlich (1986) Random surfaces in Statistical Mechanics - roughening, rounding, wetting J. Stat. Phys. 42 (5/6), 743-798

[6] F. Cesi, F. Martinelli (1996) On the layering transition of an SOS surface interacting with a wall. I. Equilibrium results. J. Stat. Phys. 82 (3/4), 823-913. 
[7] J.-D. Deuschel (1996) Entropic repulsion of the lattice free fi eld. II. The 0-boundary case. Comm. Math. Phys. 181 (3), 647-665.

[8] J.-D. Deuschel, G. Giacomin (1999) Entropic repulsion for the free fi eld: pathwise characterization in $d \geq 3$. Comm. Math. Phys. 206 (2), 447-462.

[9] E. Dinaburg, A.E. Mazel (1994) Layering transition in SOS model with external magnetic-fi eld. J. Stat. Phys. 74 (3/4), 533-563.

[10] F.M. Dunlop, P.A. Ferrari, L.R.G. Fontes (2002) A dynamic one-dimensional interface interacting with a wall. J. Statist. Phys. 107 (3/4), 705-727.

[11] P.A. Ferrari, L.R.G. Fontes, B. Niederhauser, M. Vachkovskaia (2003) The serial harness interacting with a wall. To appear in: Stochastic Process. Appl.

[12] P. A. Ferrari, S. Martínez (1998) Hamiltonians on random walk trajectories. Stochastic Process. Appl. 78 (1), 47-68.

[13] L.R.G. Fontes, M. Vachkovskaia, A. Yambartsev, in progress.

[14] J. M. Hammersley (1965/66) Harnesses. Proc. Fifth Berkeley Sympos. Mathematical Statistics and Probability, Vol. III, 89-117.

[15] P. Holický, M. Zahradník (1993) On entropic repulsion in low temperature Ising models. Cellular automata and cooperative systems (Les Houches, 1992), 275-287, NATO Adv. Sci. Inst. Ser. C Math. Phys. Sci., 396, Kluwer Acad. Publ., Dordrecht.

[16] F. den Hollander, M.V. Menshikov, S. Volkov (1995) Two problems about random walks in a random fi eld of traps. Markov Process. Related Fields 1 (2), 185-202.

[17] J.L. Lebowitz, C. Maes (1987) The effect of an external fi eld on an interface, entropic repulsion. J. Stat. Phys. 46 (1/2), 39-49.

[18] R. Pemantle, S. Volkov (1998) Markov chains in a fi eld of traps. J. Theoret. Probab. 11 (2), 561-569.

[19] F. Spitzer (1976) Principles of random walk. Springer-Verlag, New York. 
